\title{
Smartphone addiction through age and gender during Italian lockdown for COVID-19
}

\author{
Pasquale Caponnetto ${ }^{1}$, Lucio Inguscio ${ }^{2}$, Sara Valeri ${ }^{4}$, Marilena Maglia ${ }^{1}$, Riccardo Polosa ${ }^{3}$, Carlo Lai ${ }^{2}$ and \\ Giuliana Mazzoni ${ }^{2}$ \\ ${ }^{1}$ Department of Educational Science, University of Catania, Catania, Italy \\ ${ }^{2}$ Department of Dynamic and Clinical Psychology, Sapienza University, Rome, Italy \\ ${ }^{3}$ Center of Excellence for the Acceleration of Harm Reduction (Coehar), University of Catania, Catania, Italy \\ ${ }^{4}$ Department of Psychology, Sapienza University Rome, Italy
}

\begin{abstract}
Background: The dependencies associated with the Internet and technologies that can be defined Dependencies 2.0, include the smartphone. During the Italian lockdown caused by COVID19 , the use of technologies has been the fundamental basis of adaptation for smart working, school and professional training, which has led to a change in the population lifestyle. Objective: The purpose of this work is to document the level of smartphone dependence that has occurred during the COVID-19 lockdown period. Material and Methods: The NoMobilePhobia-Questionnaire (NMP-Q) was presented online to 1264 participants between the ages of 15 and 60 . Results: The results show no significant main effects for the two factors taken into account (Gender and Age of participants). Howeverthe significant interaction shows that female participants reported on average higher scores on NoMobilePhobia-Questionnaire (NMP-Q) than males, for the younger age group (15 to 44), while for those over the age of 44 , the average highest scores were for male participants. Conclusions: One of the "positive" aspects of the COVID-19 pandemic is the use of the Internet and smartphones, and our analysis aimed to document the frequency of use in the Italian context with the NoMobilePhobia-Questionnaire. The results reveal the risk in some parts of the Italian population to develop forms of smartphone dependence, especially in circumstances that prohibit direct social interactions.
\end{abstract}

Publisher's Note: MDPI stays neutral with regard to jurisdictional claims in published maps and institutional affiliations.

\section{(c) (i)}

Copyright: (c) 2020 by the authors. Submitted for possible open access publication under the terms and conditions of the Creative Commons Attribution (CC BY) license (http://creativecommons.org/licenses /by/4.0/). 